\title{
Epilepsy Surgery \\ Should Not Be a Last Resort
}

\author{
By Jack M. Gorman, MD
}

The standard treatment for epilepsy is anticonvulsant medications. Most patients respond to medical therapy with a reduction and even elimination of seizures. These drugs are generally tolerated well-enough by patients with epilepsy to permit a reasonably preserved quality of life, although often with significant adverse effects. I do not believe that the distinguished guest editor of this month's CNS Spectrums, Jerome Engel, Jr., MD, PhD, or the authors of the five excellent papers he has culled would disagree in any fundamental way with the above assertions. Nevertheless, these authors are advocates of surgical approaches to patients for whom anticonvulsant medication either does not work or is not tolerable. The authors argue that surgical resection of epileptic foci in the brain is an underutilized approach to refractory illness, that patients who are denied such care suffer unnecessarily, and that, for the most part, surgical treatment is safe. Are these reasonable assertions?

Much has been written recently about the need to disclose financial conflicts of interest and there have been sensational articles in the lay press about failures to do so. The usual case is an investigator or medical lecturer who studies or lectures about a certain medication and fails to disclose a financial connection with the drug's manufacturer. Increasingly rigorous steps are being taken by medical journals and medical schools to police this problem. At CNS Spectrums, we firmly believe in the need to disclose financial conflicts of interest and enforce that policy as vigorously as we can.

But the present case does not involve a financial conflict of interest. No medication or drug company is involved. Rather, these physicians are enthusiastic. I believe that is what makes this issue of CNS Spectrums so compelling. Surgical approaches to any neurological or psychiatric condition seem severe. Psychosurgery, when first introduced, proved disastrous and, although modern surgical approaches for illnesses like severe, refractory obsessive-compulsive disorder appear both safe and effective, the field has not yet fully recovered its standing with the public or medical profession. Surgery for neurological diseases, such as brain cancer and aneurysm, is clearly accepted, but surgery for epilepsy may strike some at first glance as a risky approach. It is important to remember, however, that current electrophysiologic and imaging technologies, often permit the precise localization of seizure foci within the central nervous system, permitting careful surgical approaches. These remedies can be dramatic in some cases, even life-saving.

What I like the most about the articles in this issue is that they represent a group of physicians willing to assemble scientific evidence in an attempt to move a field in a particular direction. Most of us are content to use accepted, traditional approaches to therapy and to be conservative even in our research. A patient with epilepsy who does not respond to anticonvulsant medication clearly needs more than that.

I believe that this month's articles may be provocative and controversial to some. I welcome and encourage letters to the editor with your thoughts, points of view, agreement, and disagreement. Bold approaches and their advocates are often what it takes to make a dent in our routine medical conservatism. CNS

\section{CALL FOR PAPERS}

CNS Spectrums is requesting submissions of case reports, reviews, and original research articles on a wide variety of neuroscientific clinical topics. Examples of topics include:

- Practical clinical psychiatry

- Neurology and neuropsychiatry in a clinical setting

- Applications of psychopharmacology and pharmacokinetics across the neuropsychiatric spectrum

Especially encouraged are papers covering comorbidities in neurologic disorders (eg, epilepsy with schizophrenia). Other crossover manuscripts geared to deepening the clinician's understanding of disorders and treatments will be given the highest priority.

All submissions are to be sent to Jack M. Gorman, MD, Editor (or, in Europe, to Joseph Zohar, MD, International Editor), c/o MBL Communications, 333 Hudson Street, 7th Floor, New York, NY 10013.

For more information, please see "Author Guidelines" on page 84 .

Dr. Gorman is the editor of this joumal and Esther and Joseph Klingenstein Professor of Psychiatry and chair of the Department of Psychiatry at Mount Sinai School of Medicine in New York City. 